\title{
LUTS durch OAB - Anticholinergika auch bei Männern eine Therapieoption
}

— Für das Auftreten von Symptomen der unteren Harnwege (LUTS) können sowohl die Prostata als auch die Blase verantwortlich sein. Frauen, die an einer überaktiven Blase $(\mathrm{OAB})$ leiden, therapiert man - anders als Männer - meist mit Anticholinergika, obwohl die irritative Symptomatik gleich ist. Männer dagegen werden eher mit Alpha-Blockern, 5- Alpha-Reduktase-Hemmern oder Phytotherapeutika behandelt. Aber auch bei Männern können Anticholinergika beim Vorliegen einer OAB die Medikamente der ersten Wahl sein. Daten zur Effektivität von Anticho- linergika, wie zum Beispiel Solifenacin (Vesikur $^{\circledR}$ ), bei Männern finden sich unter anderem in einer Metaanalyse von Chapple et al.: Unter Solifenacin 5/10 mg reduzierten sich im Vergleich zu Placebo die Anzahl der HarndrangEpisoden/24h, der Inkontinenz-Episoden/ 24 h, der Nykturie-Episoden/24 h und die Miktionsfrequenz signifikant. Das Miktionsvolumen nahm unter Solifenacin stärker zu als unter Placebo.

Ein Grund für den geringen Einsatz von Anticholinergika bei Männern ist die Befürchtung, dass sich Restharn bildet und/oder ein akuter
Harnverhalt auftritt. In einer Metaanalyse von van Kerrebroeck lag das Harnverhalt-Risiko unter Solifenacin $5 \mathrm{mg}$ allerdings bei $0 \%$ (Placebo: 0,9\%, Solifenacin $10 \mathrm{mg}:$ 0,8\%).

Die neuen EAU-Leitlinien empfehlen Anticholinergika wie Solifenacin als Alternative zu Alpha-Blockern - entweder als Monotherapie oder in Kombination mit Alpha-Blockern (Evidenzstufe 1b, Empfehlungsgrad B). Mehrere Untersuchungen zur Mono- und Kombinationstherapie stützen diese Empfehlung.

Nach Informationen von Astellas, München

\section{Längeres PSA-progressionsfreies Überleben unter GnRH-Blockern}

? Herr Prof. Miller, was hat sich in der Hormontherapie des Prostatakarzinoms in den letzten Jahren getan?

Miller: Die Hormontherapie mittels LHRHAgonisten hat sich über Jahre etabliert. Trotz kleinerer Fortschritte und Rückschläge in der Forschung sind die GnRH-Antagonisten eine der wesentlichen Entwicklungen der letzten Jahre im Konzept der Hormontherapie. Zunächst senken GnRH-Blocker wie Firmagon ${ }^{\circ}$ mit dem Wirkstoff Degarelix den Testosteronwert ebenso wie LHRH-Agonisten unter das Kastrationsniveau von $0,5 \mathrm{ng} / \mathrm{ml}$. Doch die GnRH-Blocker senken den Testosteronwert schneller und ohne initialen Anstieg, auch kommt es nicht zu kurzfristigen Testosteronerhöhungen. Hierbei handelt es sich um Phänomene, die mit negativer Tumorbiologie assoziiert sind. Aufschlussreich sind auch die Daten von Pickles et al., die auf dem diesjährigen AUA in Washington vorgestellt wurden. Die Arbeitsgruppe zeigte, dass Testosterondurchbrüche unter LHRH-Agonisten mit regelmäßiger Häufigkeit auftreten. Je nachdem, wie das Kastrationsniveau definiert wurde, sind sie bei 3-27\% der Patienten zu beobachten. Besonders häufig ist das Phänomen bei adipösen und jüngeren Patienten.

? Welche Schlüsse lassen sich aus diesen Ergebnisse ziehen?

Miller: Extensionsstudien der Zulassungsstudie von Degarelix liefern uns Hinweise, dass GnRH-Blocker möglicherweise Vorteile gegenüber LHRH-Agonisten haben. In Hinblick auf das PSA-progressionsfreie Überleben scheint
Degarelix Leuprorelin überlegen zu sein. Definiert ist das PSA-progressionsfreie Überleben durch zwei konsekutive PSA-Wert-Anstiege von $50 \%$ im Vergleich zum Nadirwert und $\geq 5 \mathrm{ng} / \mathrm{ml}$ in zwei aufeinanderfolgenden Messungen im Abstand von mindestens zwei Wochen oder Tod. Auch wenn die Studien nicht auf diese Fragestellung ausgelegt wurden, zeigen die aktuellen Vier-Jahres-Daten, dass die Patienten unter Degarelix eine signifikant längere Zeit des PSA-progressionsfreien Überlebens haben als in der Leuprorelin-Gruppe.

? Welche Mechanismen könnten dafür verantwortlich sein?

Miller: Aktuelle Daten zeigen, dass Hormone wie das FSH die Tumorprogression beeinflussen könnten. FSH-Rezeptoren wurden sowohl auf dem Prostatakarzinom als auch in den Tumorblutgefäßen gefunden. Die FSH-Rezeptorendichte ist an der Tumorgrenzfläche am höchsten. Möglicherweise ist die Expression von FSH-Rezeptoren mit einer Proliferation der Zellen an dieser Stelle verbunden. Auch scheinen FSH-Rezeptoren die Angiogenese zu stimulieren.

Betrachtet man die Wirkung der LHRH-Agonisten und GnRH-Blocker im Detail, dann führen die Blocker dazu, dass bestimmte Gonadotropine wie das FSH deutlich tiefer, konsistenter und kontinuierlicher sinken als unter Agonisten. In einer Studie von Crawford et al. fiel FSH im Degarelix-Arm um 88,5\%, unter Leuprorelin um 45,6\%. Auch wurde unter Degarelix kein Wiederanstieg von FSH beobachtet. Insgesamt sind diese Daten noch

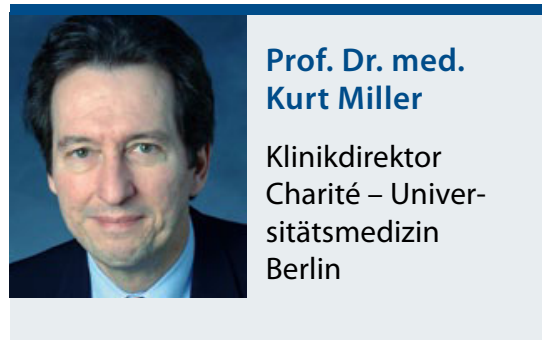

experimentell, doch geben sie Hinweise für ein Erklärungsmodell, warum die Antagonisten besser sein könnten als die Agonisten. Letztendlich warten wir darauf, dass sich die Daten in weiteren Studien bestätigen.

? In Bezug aufdie Lebensqualität der Patienten ist die Kontrolle von Knochenmetastasen ein wichtiger Aspekt. Welche Möglichkeiten bestehen in der Behandlung?

Miller: Ein Zusatznutzen der FSH-Unterdrückung, den wir bei GnRH-Blockern fanden, könnte eine protektive Wirkung niedriger FSH-Werte sein. Unter LHRH-Agonisten wurde bereits nach einem Jahr eine verminderte Knochendichte beobachtet. Und dieser Nebeneffekt ist offensichtlich viel weniger ausgeprägt, wenn das FSH-Niveau niedrig ist. Auch dieses Ergebnis gilt es durch weitere Studien zu erhärten. Ein Patient kann von niedrigen FSH-Werten profitieren, wenn er erstens nicht unter Knochendichteverlust leidet und zweitens hierfür keine Zusatzmedikamente einnehmen müsste.

Nach Informationen von Ferring, Kiel 